\title{
IL-12 gene therapy for cancer: in synergy with other immunotherapies
}

\author{
Ignacio Melero, Guillermo Mazzolini, Iñigo Narvaiza, Cheng Qian, Lieping Chen and \\ J esús Prieto
}

\begin{abstract}
In preclinical models of cancer, gene therapy with interleukin 12 (IL-12) has reached unprecedented levels of success when combined with immunotherapy approaches such as gene transfer of other cytokines and/or chemokines, costimulatory molecules or adoptive cell therapy. These combinations have been found to produce synergistic rather than additive effects. Meanwhile, IL-12 gene therapy is beginning clinical testing as a single agent, but combination strategies are at hand.
\end{abstract}

Sincethe discovery of interleukin 12 (I L-12), studies havetried to ascertain its efficacy as an anticancer agent ${ }^{1}$. Indeed, recombinant I L-12 given systemically had powerful antitumor activities in murine models that led to its clinical development, which began in 1994. I nterferon $\gamma(\mathrm{IFN}-\gamma)$ was identified as the key downstream factor induced by IL-12 that is known to be necessary for the antitumor effects. In Phasel dinical trials designed in an intrapatient dose escalation fashion, it was not realized that initial low doses of I L-12 resulted in desensitization against the biological effects of IF N- $\gamma$ produced upon subsequent I L-12 doses. I ntravenous (i.v.) doses, which weretolerated in Phasel, caused severetoxicity in Phasell clinical trials and thus put a stop to the clinical devel opment of this activeantitumoral agent for several years.

\section{Cancer gene therapy with IL-12}

When considering a protein with antitumor activity but serious systemic potential toxicity, the concept of local gene therapy comes to mind. Several groups realized simultaneously that in vivo gene transduction of malignant cells with IL-12 genes could betherapeutically useful, and would avoid toxicity by confining the potentially damaging agent to thetumor milieu. In fact, intense effects in mice were reported when using direct intratumor injection of defective recombinant adenoviruses encoding I L-12 and these vectors arestill the most powerful at achieving tumor cell genetransfer in vivo?.

All theavailable data have been generated with transplantablecell lines that represent various carcinomas, sarcomas, melanomas and lymphomas but not with spontaneously arising tumors. Generally, I L-12 genetransfer leads tosignificant antitumor activity in these experimental studies and complete eradications are often reported, but there is still room for improvement. Moreover, efficacy on naturally occurring malignancies could beless than in transplanted tumors.

Several mechanisms of antitumor activity have been identified and each contributed differently to the overall therapeutic outcome in each given tumor model. On the one hand, IL-12 promotes a potent cellular immune response in which tumor-specific cytotoxic $T$ lymphocytes (CTLs) and T hel per (Th) cells secrete Th1 cytokines. However, CD4 ${ }^{+}$Th cells are necessary for the therapeuticactivity only in some models and they seem suppressive in some instances. On the other hand, absence of CD8 ${ }^{+} \mathrm{T}$ cells severely impairs the antitumor effects in most models. Natural killer (NK)-cell depletion demonstrated a clear rolefor this lymphoid population in precisely those tumors considered less immunogenic.

IL-12 nonimmunemechanisms have been identified and themost prominent is theability of I L-12 to downregulatethe formation of new vessels into growing tumors ${ }^{3}$, also confirmed with gene transfer of IL-12 (Ref. 4). I L-12 receptors have not been found on endothel ial cells and angiogenesis is turned off by secondary mediators of the I L-12-IF N- $\gamma$ pathway, in which the $C X C$ chemokines IFN- $\gamma$-inducibleprotein 10 (IP-10) and monokine induced by IFN- $\gamma$ (Mig) have been shown to play a crucial role $e^{3}$. In addition, this cytokine cascade induces homing receptors on endothel ium for the infiltration of lymphocytes into the malignant tissue(G. Mazzolini \& al., unpublished).

\section{Cytokines and chemokines that show synergy with IL-12 gene transfer} Based on previous data with purified proteins, F.L. Graham and colleagues pioneered the combination of two adenoviruses, one encoding I L- 2 and the other IL-12, which were coinjected into tumor nodules. As a result of synergistic effects, they observed $>60 \%$ complete regression of established mammary carcinomas and induction of potent antitumor CTL activity ${ }^{5}$. Recent data show that the closely related cytokine I L-15 synergizes with I L-12, as seen with stabledouble-transfected human lung cancer cells xenografted in nudemice ${ }^{6}$. The antitumor mechanisms studied in this artificial system are independent of $\mathrm{T}$, $B$ and NK cells but unravel theintriguing involvement of neutrophils in the rejections.

IL-18 has been identified as a potent inducer of I F N $-\gamma$. I mportantly, I L-18 upregulates the expression of IL-12 receptors. In a reported poorly immunogenic tumor (MCA205), a clear synergy was observed in the antitumor effects mainly mediated by NK cells in this case $^{7}$. However, theadministration of recombinant proteins has a threatening synergistic effect in that it induces lethal levels of IF N- $\gamma$.

Genes encoding lymphocyte attracting chemokines can besuccessfully combined with IL-12. This approach has shown remarkable efficacy with adenoviruses encoding the chemokines lymphotactin 8 and IP-10 (Ref. 9). The lymphotactin gene and both IL-12 genes were engineered in thesame construct, but further research is needed to address whether a single viral vector or a combination of two vectors is 


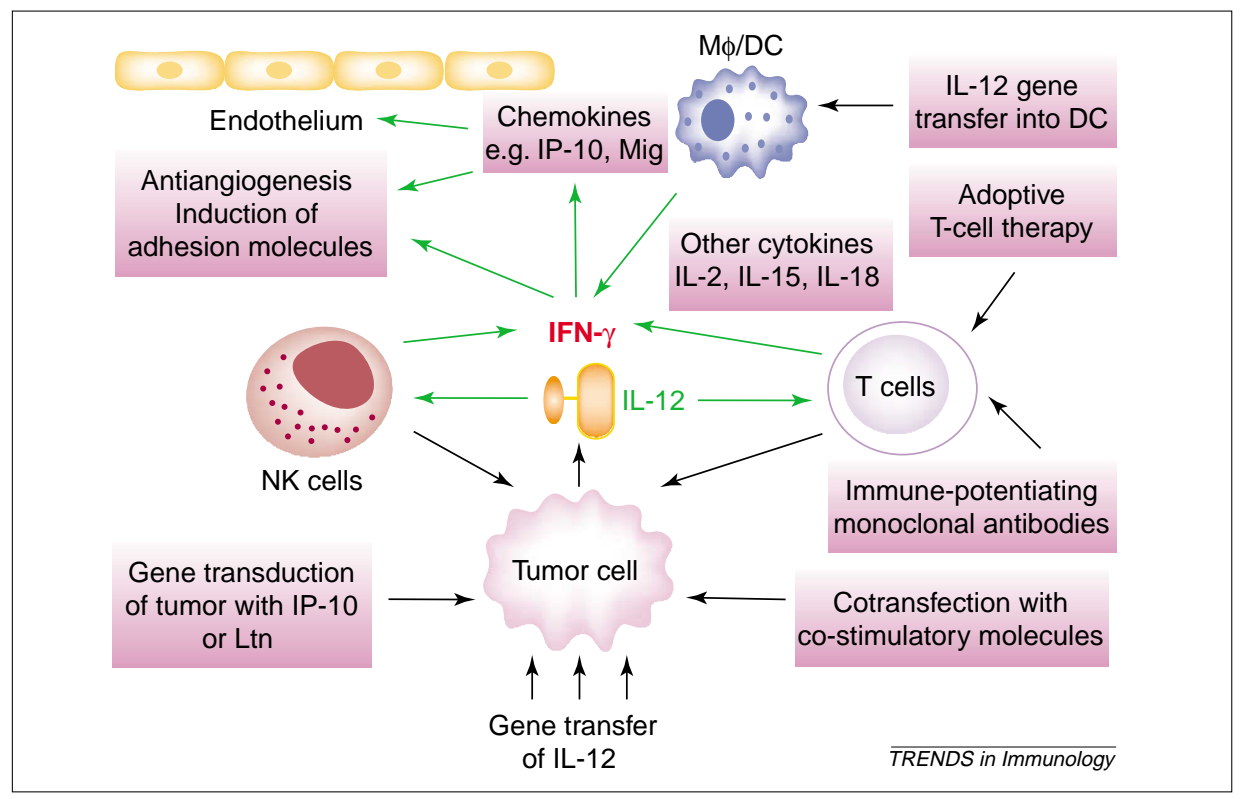

Fig. 1. Mechanisms involved in the antitumoral effects of interleukin (IL)-12 that can be potentiated in combination immunotherapy regimes as indicated in the colored boxes. IL-12 is secreted locally by transfected malignant cells and it triggers IFN- $\gamma$ production from different cellular sources. As a result, immune and nonimmune therapeutic mechanisms are turned on. The combination of IL- 12 with other immunostimulating genes or adoptive T-cell therapy has been observed to enhance efficacy in a synergistic fashion. Abbreviations: DC, dendritic cell; IFN- $\gamma$, interferon $\gamma$; IL-12, interleukin 12; IP-10, IFN- $\gamma$-inducible protein 10; Ltn, lymphotactin; Mig, monokine induced by IFN- $\gamma$; M $\phi$, macrophage; NK, natural killer.

better. Lymphotactin by itself induced lymphoid infiltration but it was dinically meaningless because mammary carcinomas progressed 8 . In addition, I P-10 transduced by adenovirus into experimental colon carcinomas had little antitumor effect but caused necrosis consistent with its antiangiogenic properties. Combination therapy with I P-10 and IL-12 showed great potency in a scenario where CD4 ${ }^{+} \mathrm{T}$ cells, $\mathrm{CD} 8^{+} \mathrm{T}$ cells and NK cells wereshowing prominent roles. Reducing the dose of the IL-12 adenovirus by one order of magnitude eradicated thetumors when combined with I P-10, thus reducing potential toxicity. Interestingly, colocalization of both adenoviruses in thesametumor nodule was required for thesystemic antitumor effect, which was not seen if given separately to two distantly implanted malignant nodules ${ }^{9}$. Other combinations are being tested, some involving three players. One important issueis toidentify a setting for comparison studies to define predinically the best regimes.

\section{Genes encoding membrane-bound costimulatory molecules}

A part from solublemediators, certain membrane-bound proteins are required to ignite and sustain theimmuneresponse.
Such molecules aretypically restricted to thesurface of professional antigenpresenting cells and can be transfected to tumors in an attempt to makethem more immunogenic.

B7 molecules are a family of proteins that interact with counter-receptors on $T$ cells and provide signals that modulate the immuneresponse. Transfection of B 7-1 (CD80) and B 7-2 (CD86) makes tumor cells more immunogenic and combination with I L- 12 has been proved beneficial in certain models ${ }^{10}$, but not in others ${ }^{11}$.

The 4-1BB ligand is a tumor necrosis factor (TNF) family member expressed on the membrane of mature dendritic cells and other cell types that upon transfection enhances the immunogenicity of tumors. This molecule interacts with 4-1BB, a surface differentiation antigen restricted to activated T cells and NK cells. Intratumor injections with adenovirus encoding 4-1BB ligand and I L-12 mutually potentiatetheir effect against large and well-established liver metastasis of an NK-sensitive colon cancer ${ }^{12}$. In addition, tumor immunity can betriggered by agonistic monoclonal antibodies (mAbs) acting on 4-1BB. Regimes of intratumor gene transfer of IL-12 and anti-4-1BB mAbs show synergizing properties ${ }^{13}$ that are more potent than those observed with the natural ligand. This is explained because antibodies show wide-reaching distribution and stimulate every available4-1BB+ lymphocyte, in contrast to the membrane bound 4-1BB natural ligand.

\section{Adoptive cell therapy strategies that synergize with IL-12 gene transfer} IL-12 induces CTL expansion, which facilitates the culture of effector cells for adoptive cell therapy regimes.

Surprisingly, I L-12 synergy with adoptive T-cell therapy not only provides CTLs, but al so operates at the effector level. These phenomena wereunveiled by studies of mice with systemictumor disease, in which somemalignant nodules were adenovirally transduced with I L-12 and subsequently given antitumor CTLsi.v. (Ref. 14). Later experimentation has shown a key role for inflammatory adhesion molecules that are induced on theendothelium of peritumoral capillaries by IL-12 gene transfer (G. Mazzolini et al., unpublished).

IL-12 can be used to upregulate artificially thefunctions of dendritic cells to promote cellular immuneresponses. Twostudies have concluded that intratumor injection of dendritic cells engineer ed to secreteI L-12, by means of adenoviral ${ }^{15}$ or retroviral transfection ${ }^{16}$, eradicates established tumors derived from at least five different tumor cell lines. IL-12 transfection into dendriticcells is probably acting at a series of levels, stimulating T cells, NK cells and dendritic cells in an autocrinefashion. Dendritic cells capture tumor antigens insidethe malignant tissueand migrate avidly to draining lymph nodes whereantigens are presented while high local levels of IL-12 arebeing produced.

\section{Conclusion}

We propose a general mechanism of action for thesesynergistic combinations in which a halt in tumor growth by angiogenesis inhibition and NK activation trailblazetheroute for artificially boosted T-cell responses (Fig. 1). Genetransfer of IL-12 into tumors is now entering dinical testing and it is hoped that the safety records will beas good as the preclinical data. Combination of the complementary immunotherapy approaches described aboveis the obvious next step to increase efficacy and minimizerisks. 


\section{Acknowledgements}

Wethank M. Duarte, E. Santiago and M.L. Subirá for suggestions and scientific discussion, and M. Mendez, J . Vidal, M.J . Huarte, CICYT and Antibióticos Farma SA for generous grants.

\footnotetext{
References

1 Trinchieri, G. (1998) Interleukin-12: a cytokineat theinterface of inflammation and immunity. Adv. Immunol. 70, 83-243

2 Bramson, J .L. \& al. (1996) Direct intratumoral injection of an adenovirus expressing interleukin-12 induces regression and longlasting immunity that is associated with highly localized expression of interleukin-12. Hum. GeneTher. 7, 1995-2002

3 Sgadari, C. et al. (1996) I nhibition of angiogenesis by interleukin-12 is mediated by the interferoninducible protein 10. Blood 87, 3877-3882

4 Barajas, M. et al. (2001) Genetherapy of orthotopic hepatocellular carcinoma in rats using adenovirus coding for IL-12. Hepatol ogy 33, 52-61

5 Addison, C.L. et al. (1998) Intratumoral coinjection of adenoviral vectors expressing IL-2 and IL-12 results in enhanced frequency of regression of injected and untreated distal tumors. GeneTher. 5, 1400-1409

6 Di Carlo, E. etal. (2000) The combined action of IL-15 and I L-12 genetransfer can inducetumor cell rejection without $\mathrm{T}$ and $\mathrm{NK}$ cell involvement. J . Immunol. 165, 3111-3118
}

7 Osaki, T. et al. (1999) Potent antitumor effects mediated by local expression of themature form of theinterferon-gamma inducing factor, interleukin-18 (IL-18). GeneTher. 6, 808-815

8 Emtage, P.C. et al. (1999) Adenoviral vectors expressing lymphotactin and interleukin 2 or lymphotactin and interleukin 12 synergize to facilitate tumor regression in murine breast cancer models. Hum. GeneTher. 10, 697-709

9 Narvaiza, l. et al . (2000) I ntratumoral coinjection of two adenoviruses, oneencoding thechemokine IFN-gamma-inducible protein-10 and another encoding IL-12, results in marked antitumoral synergy. J . I mmunol. 164, 3112-3122

10 Zitvogel, L . et al. (1996) Interleukin-12 and B 7.1 co-stimulation cooperatein the induction of effective antitumor immunity and therapy of established tumors. Eur. J . I mmunol. 26, 1335-1341

11 Sun, Y. et al. (2000) Genetransfer toliver cancer cells of B 7-1 plus interleukin 12 changes immunoeffector mechanisms and suppresses hel per T cell type 1 cytokine production induced by interleukin 12 alone. Hum. GeneTher. 11 , 127-138

12 Martinet, O. et al. (2000) I mmunomodulatory genetherapy with interleukin 12 and 4-1BB ligand: long-term remission of liver metastases in a mouse model. J . Natl. Cancer I nst. 92, 931-936

13 Chen, S.H. et al. (2000) Rejection of disseminated metastases of col on carcinoma by synergism of
IL-12 genetherapy and 4-1BB costimulation. Mol Ther. 2, 39-46

14 Mazzolini, G. etal. (2000) Adenoviral gene transfer of interleukin 12 intotumors synergizes with adoptive $T$ cell therapy both at theinduction and effector level. Hum. GeneTher. 11, 113-125

15 Melero, I. etal. (1999) Intratumoral injection of bone-marrow derived dendritic cells engineered to produce interleukin-12 induces complete regression of established murinetransplantable col on adenocarcinomas. GeneTher. 6, 1779-1784

16 Nishioka, Y. et al. (1999) Induction of systemic and therapeutic antitumor immunity using intratumoral injection of dendritic cells genetically modified to express interleukin 12 . Cancer Res. 59, 4035-4041

\section{Ignacio Melero*}

Guillermo Mazzolini

Iñigo Narvaiza

Cheng Qian

J esús Prieto

The Gene Therapy Division, Dept of

Medicine, University of Navarra,

C/Irunlarrea 1, 31008 Pamplona, Spain.

*e-mail: imelero@unav.es

\section{Lieping Chen}

Dept of Immunology, Mayo Clinic, Rochester, MN 55905, USA.

\title{
Is TGF- $\beta 1$ the key to suppression of human asthma?
}

\author{
Atsuhito Nakao
}

\section{Transforming growth factor $\beta 1$ (TGF- $\beta 1$ ) is produced by many types of cells that are activated in the asthmatic response. Recent studies have highlighted this cytokine as an important negative regulator in an experimental model of asthma. Although the role of TGF- $\beta 1$ in human asthma remains obscure, data derived from animal models have encouraged the further investigation of such suppression mechanisms in order to develop novel therapies for asthma.}

Asthma is a complex disorder characterized by airway hyperresponsiveness (AHR) and airway inflammation. Evidencehas accumulated regarding factors that promote the asthma phenotype ${ }^{1}$, but the mechanisms by which theasthma phenotypeis suppressed arelargely unclear. Recently, a pleiotropic cytokine, transforming growth factor $\beta 1$ (TGF- $\beta 1$ ), has been reported to function as a negative regulator of $A H R$ and airway inflammation in an experimental model of asthma ${ }^{2-5}$. Here, we consider this evidence and discuss possibleroles of TGF- $\beta 1$ in suppression of human asthma.

\section{Current understanding of the} pathophysiology of asthma

Asthma is a complex disorder consisting of various cellular and/or cytokine/chemokinenetworks ${ }^{1}$. The presentation of inhaled allergens to CD4 ${ }^{+}$ $T$ cells in thelungs of susceptible individuals results in the production of cytokines such as interleukin 4 (IL-4), I L-5, IL-9 and IL-13, which orchestrate the differentiation, recruitment and activation of eosinophils and mast cells in theairway mucosa ${ }^{1,6,7}$. Such effector cells releaseinflammatory mediators that causeacutebronchial constriction, disruption of theairway epithelial layer, alterations in neural control of airway tone, increased mucus production and increased smooth musclemass. These consequences of theinflammatory process induce AHR. Recent studies have shown that not only $T$ cells but also a variety of other cells, including mast cells, bronchial epithelial cells and smooth muscle cells, which are activated by various mediators, contributeto the devel opment of AHR in part through thesecretion of cytokines or chemokines that generatetissue inflammation ${ }^{8,9}$.

How does TGF- $\beta$ elicit its biological effects? TGF- $\beta 1$ is a member of the TGF- $\beta$ superfamily, which comprises a large number of cytokines ind uding TGF - $\beta$ s, bone mor phogenetic proteins (BMPs) and activins. Thesecytokines carry out a wide range of biological functions including cell proliferation, differentiation and apoptosis.

TGF - $\beta 1$ is inhibitory for inflammatory cells such as T cells, B cells, dendritic cells, mast cells and eosinophils, and also modifies the functions of structural cells such as bronchial epithelial cells, fibroblasts and bronchial smooth muscle 